\title{
CDX2 expression is progressively decreased in human gastric intestinal metaplasia, dysplasia and cancer
}

\author{
Qiang Liu ${ }^{1}$, Ming $\mathrm{Teh}^{2}$, Kosei Ito ${ }^{1,3}$, Nilesh Shah ${ }^{1}$, Yoshiaki Ito ${ }^{1,3}$ and Khay Guan Yeoh ${ }^{4}$ \\ ${ }^{1}$ Oncology Research Institute, Yong Loo Lin School of Medicine, National University of Singapore, Singapore; \\ ${ }^{2}$ Department of Pathology, Yong Loo Lin School of Medicine, National University of Singapore, Singapore; \\ ${ }^{3}$ Institute of Molecular and Cell Biology, Singapore and ${ }^{4}$ Department of Medicine, Yong Loo Lin School of \\ Medicine, National University of Singapore, Singapore
}

\begin{abstract}
Intestinal metaplasia is a key event in multistep gastric carcinogenesis. CDX2, a master regulator of intestinal phenotype, was shown to play a tumor-suppressive role in colon cancer. However, it was reported to be expressed in nearly all gastric intestinal metaplasia and many gastric cancers. As CDX2 is differentially expressed in normal stomach and intestine, we sought to relate the CDX2 expression to gastrointestinal differentiation along gastric carcinogenesis. The expression of CDX2 protein in gastric intestinal metaplasia, dysplasia and cancer was examined and related to their gastrointestinal differentiation. CDX2 expression was significantly decreased in incomplete intestinal metaplasia, which expresses both gastric mucins (MUC5AC and MUC6) and intestinal mucin (MUC2), compared with complete intestinal metaplasia, which expresses intestinal mucin (MUC2) only. Although incomplete intestinal metaplasia morphologically resembles colon, its CDX2 expression was apparently lower than that in the normal colon. Moreover, CDX2 expression was progressively reduced in gastric dysplasia and cancer. The CDX2 expression in gastric cancer was also inversely correlated with the expression of gastric mucins. As incomplete intestinal metaplasia is associated with higher risk of gastric cancer, its lower CDX2 expression compared with that in complete intestinal metaplasia and normal colon epithelium resolved the current contradiction between the tumor-suppressive role of CDX2 in the colon and the high prevalence of CDX2 in intestinal metaplasia. Further decrease of CDX2 expression in gastric dysplasia and cancer suggests that CDX2 plays a similar anticarcinogenic role in intestinal metaplasia as it does in colon. Intestinal metaplasia or dysplasia with low expression of CDX2 may serve as predictive markers for gastric cancer.
\end{abstract}

Modern Pathology (2007) 20, 1286-1297; doi:10.1038/modpathol.3800968; published online 28 September 2007

Keywords: gastric cancer; gastric dysplasia; intestinal metaplasia; CDX2

It was proposed by Correa $^{1}$ that human gastric carcinogenesis is a multistep process that progresses from chronic gastritis, atrophy, intestinal metaplasia (IM), dysplasia and finally leads to gastric cancer. This model, although challenged by a few, ${ }^{2,3}$ is widely accepted, especially for the intestinal type of gastric cancer. ${ }^{4}$ IM in the stomach, a key event in gastric carcinogenesis, is the replacement of gastric mucosa by an epithelium that histologically resembles intestinal mucosa. However, IM is present in

Correspondence: Dr KG Yeoh, MBBS, MMed (Int Med), FRCP, FRCP (Glasg), FAMS, Department of Medicine, Yong Loo Lin School of Medicine, National University of Singapore, 10 Medical Drive, Singapore 117597, Singapore.

E-mail: mdcykg@nus.edu.sg

Received 4 April 2007; accepted 16 July 2007; published online 28 September 2007 about $20 \%$ of all gastric biopsies ${ }^{5}$ and only a minority will progress to gastric cancer. IM is also present in the antrum with duodenal ulcer, which is associated with lower risk of gastric cancer. ${ }^{6}$ Thus, the predictive value of IM as a marker for the risk of developing gastric cancer is very low.

IM can be classified into different subtypes by several classification systems. The most widely accepted one is to classify IM into complete type and incomplete type, with the latter carrying a higher risk of gastric cancer. ${ }^{7}$ The complete type is characterized by the presence of absorptive cells, Paneth cells and goblet cells secreting sialomucins, similar to the small intestinal phenotype. The incomplete type is characterized by the presence of columnar and goblet cells secreting sialomucins and/or sulphomucins, similar to the colonic phenotype. Using alcian-blue/periodic acid Schiff and 
high-iron diamine-alcian blue technique, Filipe and Jass $^{8}$ classified IM into three subtypes. Type I corresponds to the complete type, while type II and type III were classified from the incomplete type according to the mucins secreted by the columnar cells: sialomucins in type II and sulphomucins in type III. Several studies claimed that only type III of IM is associated with an increased risk of gastric cancer, ${ }^{9-11}$ but other reports cast doubt on it. ${ }^{12-14}$ Different subtypes of IM may coexist in the same foci, and this mosaic pattern complicates efforts at subtyping. ${ }^{14}$

CDX2, a caudal-related homeobox transcription factor, is well known for its function in the development and maintenance of intestinal epithelium. In normal adults, CDX2 is expressed in the small and large intestines but not in the stomach and esophagus. Although a recent report showed the increase of CDX2 mRNA and protein in human colorectal cancer, ${ }^{15}$ several studies have demonstrated that CDX2 mRNA and protein was reduced or lost in human colorectal cancer. ${ }^{16-18}$ Exogenous CDX2 expression in colon cancer cell lines inhibited the proliferation, invasion and migration of cancer cells while promoting the expression of genes characteristic of mature enterocytes. ${ }^{19,20}$ It was reported that mice doubly heterozygous for APC ${ }^{\Delta 716}$ and $\mathrm{Cdx} 2^{+/-}$develop six times more colonic polyps than their $\mathrm{APC}^{\Delta 716}$ and $\mathrm{Cdx} 2^{+/+}$littermates. ${ }^{21}$ Furthermore, the carcinogen azoxymethane induced invasive adenocarcinoma of the distal colon in $\mathrm{Cdx} 2^{+1-}$ mice but not in wild-type littermates. ${ }^{22}$

CDX2 expression has also been implicated in gastric carcinogenesis. However, the role of CDX2 in gastric carcinogenesis is controversial. Using $\mathrm{Cdx} 2$ transgenic mice, two groups have reported that the expression of $\mathrm{Cdx} 2$ transformed the gastric mucosa into intestinal metaplastic Mucosa, ${ }^{23,24}$ and in one model it progressed to gastric cancer. ${ }^{25}$ Several studies showed CDX2 expression in nearly all IM and many gastric cancers, suggesting that ectopic CDX2 expression is involved in gastric carcinogenesis. ${ }^{26-30}$ It was also reported that the disruption of CDX2 did not affect the tumorigenic potential in a gastric cancer cell line. ${ }^{31}$ On the other hand, most of the studies so far have demonstrated that positive CDX2 expression in gastric cancer was significantly correlated with better differentiation and prognosis. ${ }^{28-30,32}$

Thus, an apparent contradiction exists in the literature. Whereas CDX2 has a tumor-suppressive role in the colon, the stomach shows a high expression of CDX2 in IM, which is considered to be a pre-neoplastic lesion. As CDX2 is physiologically expressed in normal intestine but not in normal stomach, we examined the expression of CDX2 protein in IM, gastric dysplasia and cancer and analyzed the relationship between the CDX2 expression and their gastrointestinal differentiation, using MUC5AC and MUC6 as markers of gastric differentiation and MUC2 as a marker of intestinal differentiation. With a finely tuned staining protocol, we found that CDX2 protein is not evenly expressed in all IM foci, but significantly decreased in incomplete IM compared with complete IM. Although incomplete IM morphologically resembles the colon, its CDX2 expression is significantly lower than that in normal colon. Moreover, the CDX2 expression is progressively reduced in gastric dysplasia and cancer, suggesting that the loss of CDX2 may have similar carcinogenic role in IM as in the colon.

\section{Materials and methods}

\section{Patients and Tissue Samples}

Surgically resected gastric adenocarcinoma samples and matching noncancerous tissues from 70 patients were obtained from the Department of Pathology and Surgery, National University of Singapore, under a protocol approved by the Institutional Review Board. There were 47 males and 23 females, aged 31-86 years (mean \pm standard error of the mean, $62.6 \pm 1.6$ ). According to Lauren's classification for gastric adenocarcinoma, 45 cases were of intestinal type and 19 cases of diffuse type. The other six cases of mucinous adenocarcinoma displayed an intestinal-type pattern of infiltration. It was listed as an individual group in this study because of its unique pattern of mucin expression. ${ }^{29}$ The 45 cases of intestinal type gastric adenocarcinoma were further classified into well differentiated (14 cases), moderately differentiated (11 cases) and poorly differentiated (20 cases), according to their morphological features.

In the cancer and matching noncancerous tissues of these 70 patients, 38 foci of IM were found in the tissues of 26 cases, including 18 IM foci just beside the cancer in 16 cases and 20 IM foci on the noncancerous tissues in 15 cases.

In addition, surgically resected colon adenocarcinoma samples with matching noncancerous tissues from five patients and 31 gastric dysplasia biopsies from 29 patients were obtained from the Department of Pathology and Surgery, National University of Singapore, under a protocol approved by the Institutional Review Board. The 31 gastric dysplasia biopsies were classified into 15 low-grade dysplasia and 16 high-grade dysplasia by experienced pathologists.

\section{Histochemistry}

The samples were fixed with $10 \%$ neutral-buffered formalin and embedded in paraffin. Paraffin-embedded samples were serially sectioned at $4 \mu \mathrm{m}$ and mounted on slides. IM was classified into complete type and incomplete type, using Alcian Blue $(\mathrm{pH}$ 2.5)/Periodic Acid-Schiff (AB/PAS) staining (Dako AB/PAS stain system, Dakocytomation, Carpinteria, 
CA, USA). After deparaffinization and rehydration, the sections were incubated with Alcian Blue $\mathrm{pH} 2.5$ for $30 \mathrm{~min}$, followed by $0.5 \%$ periodic acid for $10 \mathrm{~min}$ and Schiff solution for $10 \mathrm{~min}$. The sections were then counterstained with modified Mayer's hematoxylin for $5 \mathrm{~min}$, dehydrated with graded ethanols and mounted with coverslip. In complete IM, only goblet cells were stained blue, while in incomplete IM both the goblet cells and the intermediate columnar cells appeared blue.

\section{Immunohistochemistry}

After routine deparaffinization and rehydration of the slides, antigen retrieval was done by incubation in modified citrate buffer (target retrieval solution, Dakocytomation, Carpinteria, CA, USA) at $96^{\circ} \mathrm{C}$ for $40 \mathrm{~min}$ (for MUC2, MUC5AC, MUC6 and Ki67 antibodies) or at $121^{\circ} \mathrm{C}$ for $20 \mathrm{~min}$ (for CDX2 antibody). The sections were treated with $0.03 \%$ hydrogen peroxide for 5 min to block the endogenous peroxidase activity, followed by incubation with anti-CDX2 monoclonal antibody (1:100, Biogenex, San Ramon, CA, USA), anti-MUC2 monoclonal antibody (1:100, Novocastra Laboratories, Newcastle, UK), anti-MUC5AC monoclonal antibody (1:100, Novocastra Laboratories, Newcastle, UK), anti-MUC6 monoclonal antibody (1:100, Novocastra Laboratories, Newcastle, UK) or anti-Ki67 monoclonal antibody (1:100, Dakocytomation, Carpinteria, CA, USA) at room temperature for 1 hour. The sections were then incubated with peroxidaselabeled polymer from Envision + System-HRP (DAB) kit (Dakocytomation, Carpinteria, CA, USA) at room temperature for $1 \mathrm{~h}$. After development with diaminobenzedine, the sections were counterstained with hematoxylin, dehydrated with graded ethanols and xylene and then mounted with coverslip.

\section{Assessment of Immunostaining in Cancer Cells}

At least 10 representative fields under high-power magnification $(\times 400)$ were chosen and 1000 cancer cells were counted for each section. If the number of cancer cells was less than 1000, then all available cancer cells were counted.

Both qualitative and semiquantitative approaches were used in scoring the staining of MUC2, MUC5AC, MUC6 and CDX2. Samples were classified as positive if $>5 \%$ tumor cells stained positive and otherwise as negative.

Semiquantitative scores were given as the score of the percentage of positive cells plus the score of the staining intensity. The scoring criteria of the percentage of positive cells were as follows: score $0,0-5 \%$ positive cancer cells; score 1, 6-25\% positive cancer cells; score $2, \quad 26-50 \%$ positive cancer cells; score 3,51-75\% positive cancer cells; score $4,76-100 \%$ positive cancer cells. The intensity score was given as follows: score 0, no staining; score 1, weak/equivocal staining; score 2, mild staining; score 3 , moderate staining; score 4, strong staining. The final scores were from 0 to 8.

Ki67 index was defined as the percentage of Ki-67-positive cancer cells.

Two experienced investigators independently examined the staining while blind to the clinicopathological data. Different scores between the two investigators were observed in $<15 \%$ of the cases, and a consensus was achieved in all the cases after discussion.

\section{Statistical Analysis}

Fisher's exact test or $\chi^{2}$ test was used to calculate the difference of distribution in two or three groups. T-test or one-way ANOVA with Bonferroni test was used to compare the means between the two groups or among the three groups. Differences were assumed to be statistically significant when $P<0.05$.

\section{Results}

The expression of MUC2, MUC5AC, MUC6 and CDX2 in Normal Gastric Mucosa and Intestinal Metaplasia

In normal gastric mucosa, MUC5AC was expressed in the cytoplasm of superficial foveolar epithelium, while MUC6 was expressed in the cytoplasm of mucous neck cells of the body and deeper glands of the antrum. MUC2 and CDX2 are not expressed in normal gastric mucosa (Table 1).

Thirty-eight foci of IM were classified into 19 foci of complete type and 19 foci of incomplete type by AB/PAS staining. MUC2 was expressed in the cytoplasm of goblet cells in all IM glands. MUC5AC was expressed in the cytoplasm of both goblet cells and columnar cells in incomplete IM, whereas it was expressed in only a few goblet cells but not absorptive cells in complete IM. MUC6 was expressed in both goblet cells and columnar cells, in several deeper glands of incomplete IM but not in complete IM (Figures 1 and 2, Table 1). This is in agreement with previous studies that showed incomplete IM with co-expression of intestinal and gastric mucins, while complete IM with de novo expression of intestinal mucin but decreased expression of gastric mucins. ${ }^{33,34}$ In a few glands within the foci of complete IM, MUC5AC and MUC6 were expressed in both goblet cells and columnar cells and this demonstrated the 'mosaic' pattern of IM subtypes (Figure 2).

CDX2 protein was strongly expressed in the nucleus of both goblet cells and absorptive cells in the foci of complete IM (score 8), but it was significantly decreased in the foci of incomplete IM (score $\sim 6$ ) (Figure 1). Furthermore, within the foci of mosaic IM, the CDX2 expression in the glands, which expressed MUC5AC or MUC6, was 
Table 1 The expression of MUC2, MUC5Ac, MUC6 and CDX2 in normal gastric mucosa, complete IM, incomplete IM and normal colonic mucosa

\begin{tabular}{|c|c|c|c|c|}
\hline & MUC2 & $M U C 5 A C$ & MUC6 & $C D X 2$ \\
\hline $\begin{array}{l}\text { Normal gastric } \\
\text { mucosa }\end{array}$ & - & $\begin{array}{l}+ \text { in superficial foveolar } \\
\text { epithelium }\end{array}$ & $\begin{array}{l}+ \text { in the mucous neck cells of } \\
\text { the body and deeper glands } \\
\text { of the antrum }\end{array}$ & - \\
\hline Complete IM & + in goblet cell & - or only + in few goblet cells & - & $\begin{array}{l}\text { Strongly positive in both } \\
\text { goblet cells and columnar } \\
\text { cells }\end{array}$ \\
\hline Incomplete IM & + in goblet cell & $\begin{array}{l}+ \text { in both goblet cells and } \\
\text { columnar cells }\end{array}$ & $\begin{array}{l}+ \text { in both goblet cells and } \\
\text { columnar cells in deeper } \\
\text { glands }\end{array}$ & $\begin{array}{l}\text { Mildly positive in both goblet } \\
\text { cells and columnar cells }\end{array}$ \\
\hline $\begin{array}{l}\text { Normal colonic } \\
\text { mucosa }\end{array}$ & + in goblet cell & - & - & $\begin{array}{l}\text { Strongly positive in every } \\
\text { cell }\end{array}$ \\
\hline
\end{tabular}

apparently lower than that in the glands that did not express MUC5AC and MUC6 (Figure 2). This indicates that the decrease of CDX2 expression is consistent at the gland level, but not at the focus level, of incomplete IM.

\section{The Expression of CDX2 in Normal Colon and Colon Cancer}

CDX2 protein was strongly expressed in the nucleus of normal colon epithelial cells, from superficial to deeper glands. Two out of the five colon cancer cases tested showed loss or significantly decreased CDX2 expression compared with normal colon epithelium (Figure 3).

\section{The Expression of MUC2, MUC5AC, MUC6 and CDX2 in Gastric Dysplasia}

The results were summarized in Table 2. Goblet cells were seen either in or surrounding the dysplastic area in 28 biopsies out of 31 gastric dysplasia biopsies, suggesting the close relationship between gastric dysplasia and IM. MUC2 was expressed in the cytoplasm of goblet cells in 22 dysplasia biopsies. Gastric mucins, MUC5AC and MUC6 were positive in 16 dysplasia biopsies. The expression of Mucins decreased from incomplete IM to dysplasia, indicating less differentiation and maturation of the cells in dysplasia. Unlike the uniform expression pattern in each gland of IM, the expression of Mucins and CDX2 varies among cells in the same dysplastic gland (Figure 4). Each gastric gland is known to be of monoclonal origin. ${ }^{35}$ Therefore, the mosaic patterns of the gastric and intestinal mucins within a single gland suggest that induction of gastric or intestinal phenotype is unstable at dysplasia and probably occurs after gland formation in dysplasia. The CDX2 expression in dysplasia (score 3.9) was further decreased compared with that in IM. Moreover, the CDX2 expression in highgrade dysplasia (score 2.75) was also significantly lower than that in low-grade dysplasia (score 5.13) $(P<0.05)$. However, the expression of MUC2,
MUC5AC and MUC6 in dysplasia was not associated with the grade of dysplasia and the CDX2 expression $(P>0.05)$.

\section{Expression of MUC2, MUC5AC, MUC6 and CDX2 in Gastric Cancer}

The results are summarized in Table 3. MUC2 protein was expressed in $100 \%$ mucinous type of gastric cancer $(6 / 6$, score $7.17 \pm 0.54)$ and was significantly higher than that in the intestinal type of gastric cancer $(23 / 45$, score $3.24 \pm 0.49)(P<0.05)$. None of the mucinous types of gastric cancer were positive for MUC6 (0/6). This is in agreement with a previous study, that identified a unique expression pattern of mucins in the mucinous type of gastric cancer. ${ }^{29}$

The CDX2 score of the gastric cancers with positive MUC2 expression was significantly higher than that of the gastric cancers with negative MUC2 expression $(P<0.01$ for intestinal-type gastric cancer and $P<0.05$ for diffuse-type gastric cancer). However, the CDX2 score of the gastric cancers with positive MUC5AC expression was significantly lower than that of the gastric cancers with negative MUC5AC expression $(P<0.05$ for both intestinal and diffuse-type gastric cancers). MUC6 also showed a similar trend as MUC5AC in gastric cancer, although the difference was statistically insignificant $(P=0.054$ for intestinal type and $P=0.289$ for diffuse type) (Figure $5 \mathrm{a}$ and $\mathrm{b}$ ). This indicates an inverse relationship between CDX2 expression and the expression of gastric mucins in gastric cancer, similar to the one in IM. Representative pictures are shown in Figure 6.

Using MUC5AC and MUC6 as the markers of gastric differentiation and MUC2 as the marker of intestinal differentiation, the gastric cancers were classified into four categories: $\mathrm{G}$ type, positive for one or both gastric mucins (MUC5AC and MUC6) only; GI type, positive for intestinal mucin (MUC2) and at least one of the gastric mucins (MUC5AC and MUC6); I type, positive for intestinal mucin (MUC2) only; N type, none of the MUC2, MUC5AC and 

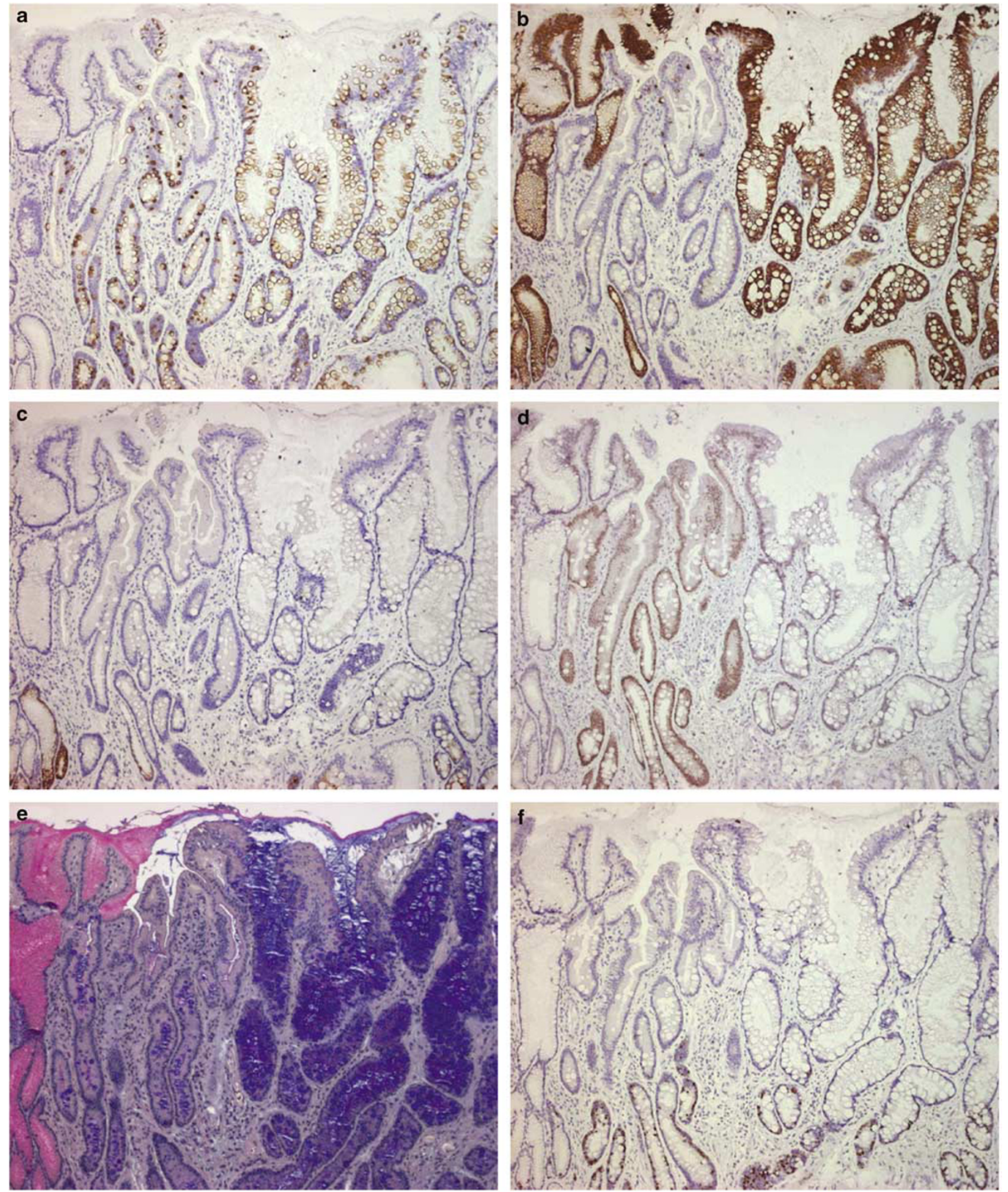

Figure 1 Immunohistochemistry staining of MUC2 (a), MUC5AC (b), MUC6 (c), CDX2 (d) and Ki67 (f) and ABPAS staining (e) in a focus of complete IM (left part) and an adjacent focus of incomplete IM (right part). Besides the presence of MUC2-positive goblet cells, incomplete IM expressed gastric mucins (MUC5AC in almost all glands and Muc6 in several deeper glands) in goblet cells and columnar cells, while complete IM only expressed MUC5AC in few goblet cells but not in the absorptive cells. The CDX2 expression was significantly decreased in the incomplete IM than that in complete IM. 

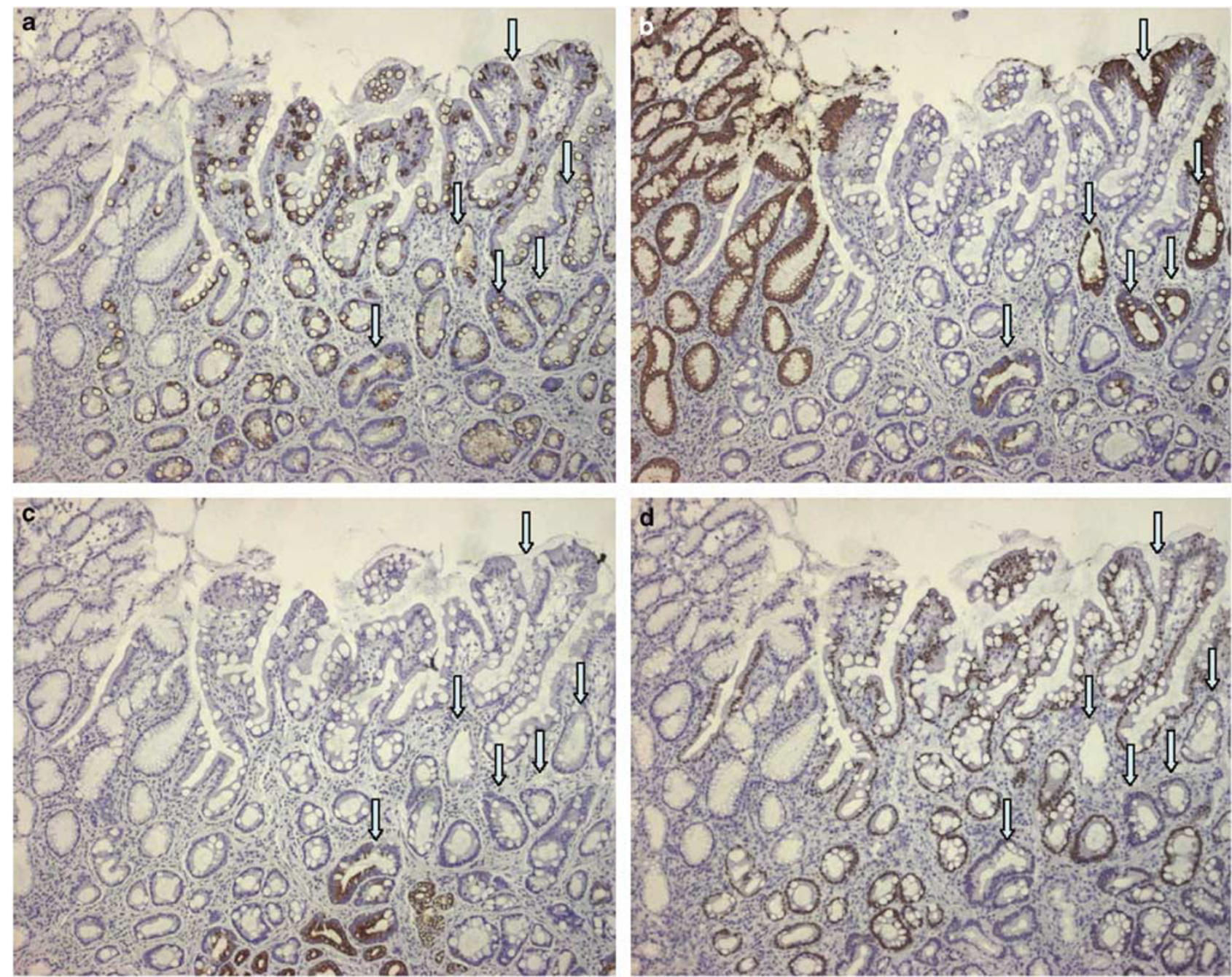

Figure 2 Immunohistochemistry staining of MUC2 (a), MUC5AC (b), MUC6 (c) and CDX2 (d) in a focus of mosaic IM. The CDX2 expression in the incomplete IM glands, which expressed both gastric mucins and intestinal mucin (the glands pointed by arrows) was significantly lower compared with other complete IM glands, which only expressed intestinal mucin.

MUC6 are positive. ${ }^{36}$ The 70 gastric cancer cases were classified into 20 cases of G type, 26 cases of GI type, 14 cases of I type and 10 cases of $\mathrm{N}$ type. The CDX2 score in I type of gastric cancer was the highest among the four groups, which is significantly higher than that in GI type of gastric cancer $(P<0.05)$. The CDX2 score in GI type was also significantly higher than that in $\mathrm{G}$ type of gastric cancer $(P<0.01)$ (Figure 5c).

\section{The Ki67 Expression in Normal Gastric Mucosa, IM and Gastric Cancer}

Ki67-positive cells were found in the neck region of normal gastric mucosa. Ki67 was also expressed in some cells in deeper glands of IM, with no significant difference between complete IM and incomplete IM. The Ki67 index of cancer was not significantly associated with the clinicopathological data and the expression of MUC2, MUC5AC, MUC6 and CDX2.

\section{Correlation of the Expression of CDX2 and Mucins with Clinicopathological Features}

The age of the patients with intestinal type of gastric cancer $(66.2 \pm 1.6)$ was significantly higher than that of the patients with diffuse type of gastric cancer $(54.6 \pm 3.7) \quad(P<0.01)$. There was no significant association between the expression of MUC2, MUC5AC, MUC6 and CDX2 and the clinicopathological data, including age and sex.

\section{Discussion}

Unlike previous studies that showed the expression of CDX2 uniformly in nearly all IM foci, this study, demonstrated for the first time, that the expression of CDX2 is significantly decreased in incomplete IM, 
which express both gastric and intestinal mucins compared with complete IM, which only express intestinal mucin. The difference between our results and those of others may be explained by the different antibodies and different staining protocols, including the lower concentration of anti-CDX2 antibody and shorter incubation time used in our
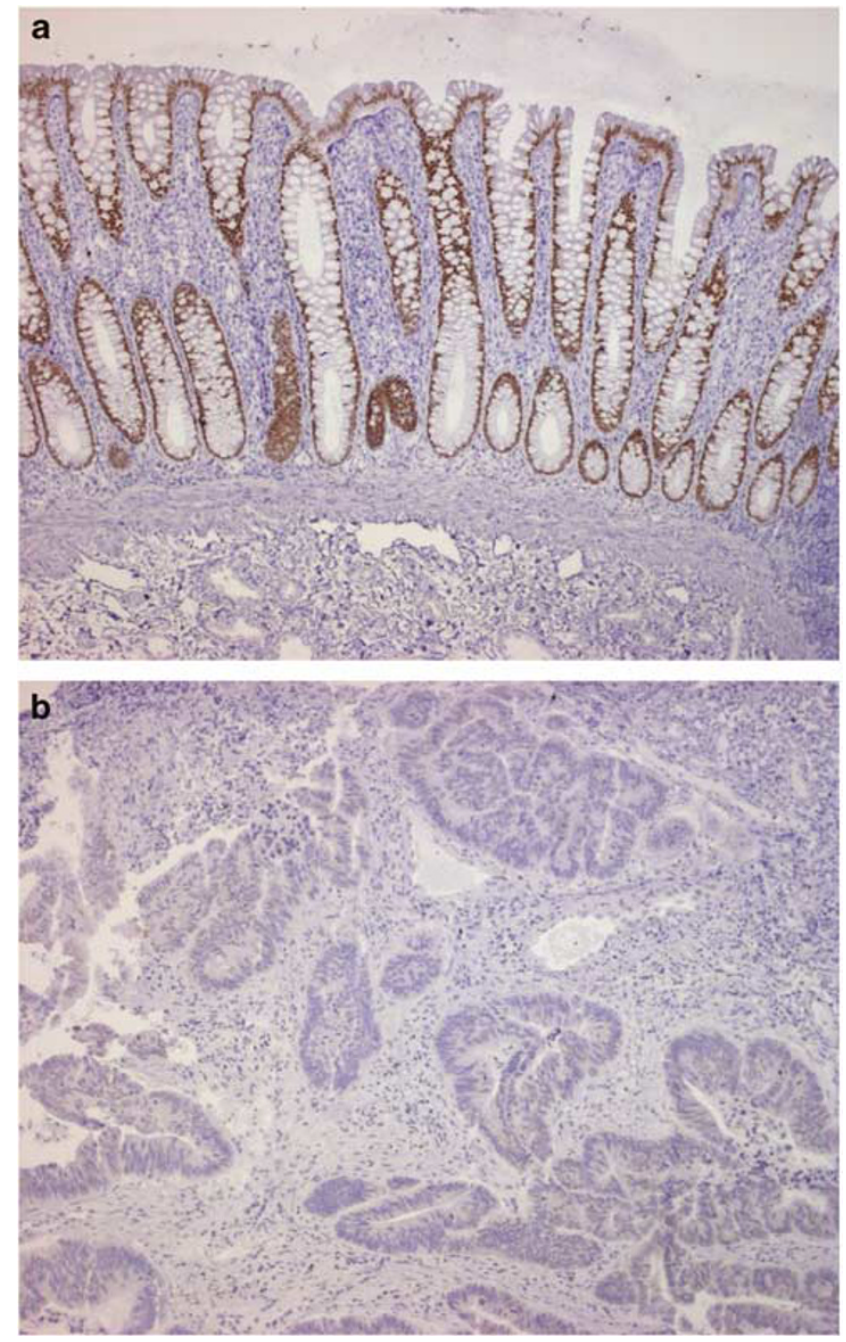

Figure 3 The CDX2 expression in colon cancer (b) was significantly decreased compared with that in normal colon (a). The tissues in A and B were from the same patient. studies. During the optimization of the CDX2 staining protocol, we found that several experimental procedures affected the final staining pattern. Higher concentrations of CDX2 antibody, excessive incubation of primary antibody or stronger antigen retrieval enhanced the CDX2 staining intensity in incomplete IM and thus diminished the difference between incomplete IM and complete IM (Data not shown).

Because mild expression of CDX2 is still observed in incomplete IM, which morphologically resembles the colon, we compared the CDX2 expression in incomplete IM with that in normal colon. CDX2 was strongly expressed in the epithelium of normal colonic mucosa and was much stronger than that in incomplete IM. Among the five colon cancer cases tested, two cases demonstrated loss or significant reduction of CDX2 protein in cancer, consistent with the previously reported tumorsuppressive role of CDX2 in colon. Considering that incomplete IM is associated with higher risk of gastric cancer than complete IM, the decrease of CDX2 in incomplete IM may be important in gastric carcinogenesis as it is in colon. Further reduction or loss of CDX2 in gastric dysplasia and gastric cancer support the tumor-suppressive role of CDX2 in IM as in the colon.

There seems to be an initial increase followed by the progressive decrease of CDX2 expression along Correa's cascade of gastric carcinogenesis (Figure 7). Various factors, such as Helicobacter pylori infection $^{37}$ and bile reflux, ${ }^{38}$ could induce ectopical CDX2 expression in normal stomach, which leads to intestinalization. Gastric mucosa may become complete IM if CDX2 expression is sufficiently strong. In this case, cells would become terminally differentiated and lose malignancy. On the other hand, incomplete IM would be induced if CDX2 expression is not strong enough. Incomplete IM would be in an unstable intermediate state. The cells in some incomplete IM may not be able to terminally differentiate into either gastric or intestinal cells. These unstable cells may have greater risk for gastric cancer because relatively low CDX2 may lead to inadequate anticarcinogenic function. Anticarcinogenic function of CDX2 may be associated with its ability to induce terminal differentiation. It is often

Table 2 The expression of MUC2, MUC5AC, MUC6 and CDX2 in gastric dysplasia

\begin{tabular}{|c|c|c|c|c|c|c|c|c|}
\hline \multirow[t]{2}{*}{ Dysplasia } & \multicolumn{2}{|c|}{ MUC2 } & \multicolumn{2}{|c|}{$M U C 5 A C$} & \multicolumn{2}{|c|}{ MUC6 } & \multicolumn{2}{|c|}{$C D X 2$} \\
\hline & $\begin{array}{c}\text { Positive cases } \\
(\%)\end{array}$ & Score ${ }^{\mathrm{a}}$ & $\begin{array}{c}\text { Positive cases } \\
\text { (\%) }\end{array}$ & Score ${ }^{\mathrm{a}}$ & $\begin{array}{c}\text { Positive cases } \\
(\%)\end{array}$ & Score $^{\mathrm{a}}$ & $\begin{array}{c}\text { Positive cases } \\
\text { (\%) }\end{array}$ & Score ${ }^{\mathrm{a}}$ \\
\hline High grade $\left(16^{\mathrm{b}}\right)$ & $9(56 \%)$ & $3.31 \pm 0.79$ & $7(44 \%)$ & $2.81 \pm 0.84$ & $3(19 \%)$ & $1.00 \pm 0.54$ & $9(56 \%)$ & $2.75 \pm 0.72^{*}$ \\
\hline Low grade (15) & $13(87 \%)$ & $5.26 \pm 0.63$ & $9(60 \%)$ & $3.80 \pm 0.87$ & $3(20 \%)$ & $1.00 \pm 0.53$ & $12(80 \%)$ & $5.13 \pm 0.74^{*}$ \\
\hline Total (31) & $22(71 \%)$ & $4.26 \pm 0.53$ & $16(52 \%)$ & $3.29 \pm 0.60$ & $6(19 \%)$ & $1.00 \pm 0.37$ & $21(68 \%)$ & $3.90 \pm 0.55$ \\
\hline
\end{tabular}

\footnotetext{
${ }^{\mathrm{a}}$ Scores were expressed as the mean \pm s.e.m.

${ }^{\mathrm{b}}$ The figures in parentheses in this column are the numbers of cases.

${ }^{*} P<0.05$ when compared with the other in the same column.
} 

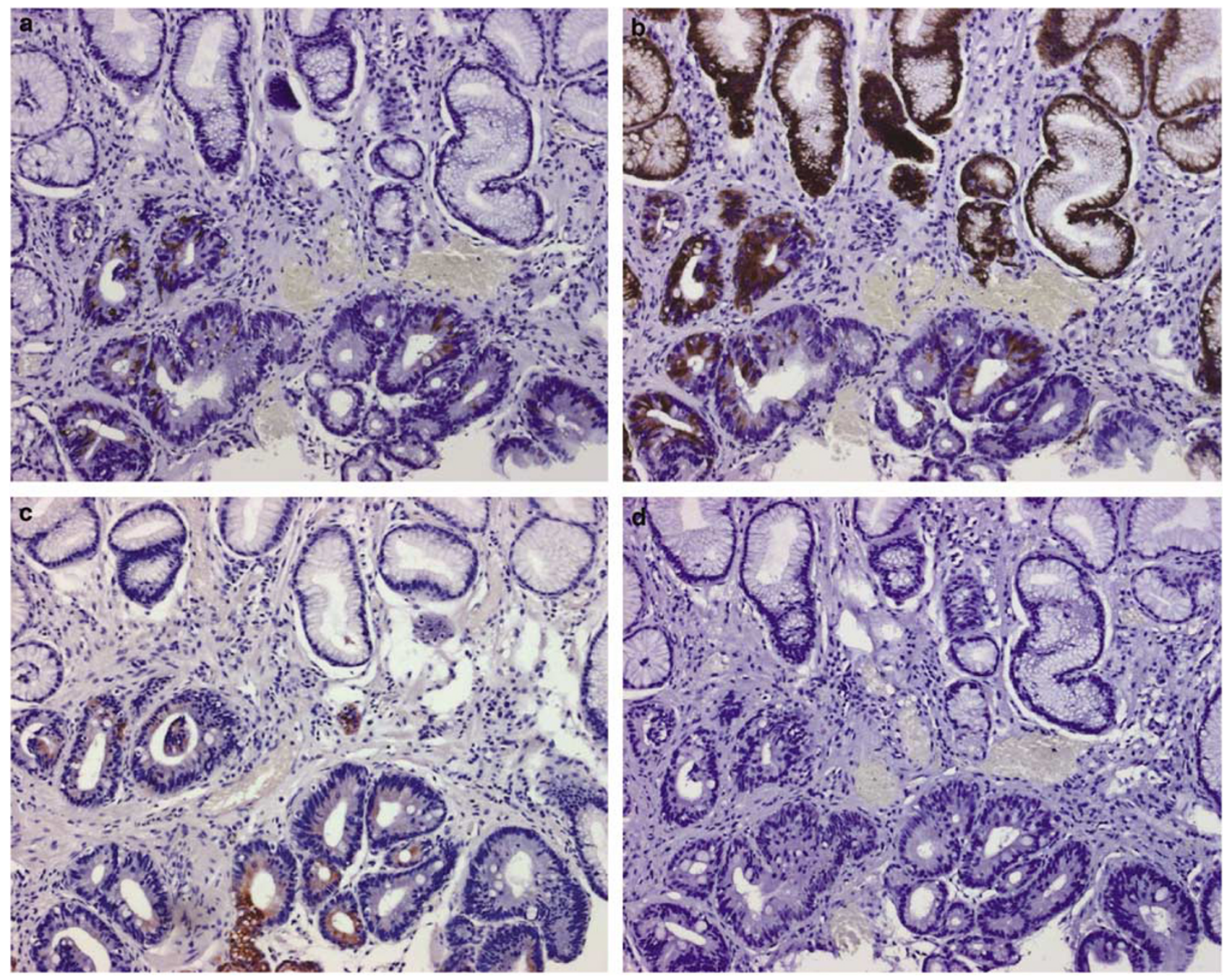

Figure 4 Immunohistochemistry staining of MUC2 (a), MUC5AC (b), MUC6 (c), CDX2 (d) in a biopsy of gastric dysplasia. The CDX2 expression was lost in the dysplastic glands, which were also incomplete IM with expression of both gastric and intestinal mucins.

observed that stronger CDX2 expression was associated with better differentiation of gastric cancer tissues ${ }^{28-30,32}$ and cell lines. ${ }^{39}$ Further decrease of CDX2 in incomplete IM may lead to a less differentiated state and more instability. This scenario may explain the observed progressive decrease of CDX2 from incomplete IM to dysplasia to cancer.

Although we showed that the expression of CDX2 is progressively decreased in incomplete IM, gastric dysplasia and cancer, the mechanisms underlying the decrease are not clear yet. The mutation and rearrangements of CDX2 gene was shown to be infrequent in colon cancer. ${ }^{40}$ Lorentz et $a{ }^{41}$ showed that the activation of PKC pathway by RAS might be responsible for the downregulation of CDX2 expression in colon cancer. Recently, the CDX2 promoter methylation was implicated in the downregulation of CDX2 in gastric cancer patients ${ }^{42}$ and colorectal cancer patients. ${ }^{43}$ Further studies of the mechanisms of CDX2 downregulation in gastric carcinogenesis are needed.
Incomplete IM itself has inadequate predictive value as a marker for high risk of gastric cancer. It is possible that further loss of CDX2 in incomplete IM could be a key step that may precede the morphological change to dysplasia. Quantitation of CDX2 expression in incomplete IM may help to predict the risk of gastric cancer.

Although IM was more associated with intestinal type of gastric cancer than diffuse type, our study showed comparably high expression of MUC2 and CDX2 in diffuse type of gastric cancer as in intestinal type. This is consistent with previous studies that suggested that intestinal differentiation markers are not exclusive to intestinal type of gastric cancer and that diffuse type of gastric cancer also can have features of intestinal differentiation in a high proportion of cases. ${ }^{26,44}$ Whether intestinalization is also involved in the carcinogenesis of diffuse type of gastric cancer is not clear yet.

In summary, this study reported the significant decrease of CDX2 expression in incomplete IM compared with complete IM and normal colon, 
Table 3 The expression of MUC2, MUC5AC, MUC6 and CDX2 in gastric cancer

\begin{tabular}{|c|c|c|c|c|c|c|c|c|c|c|}
\hline \multicolumn{3}{|c|}{ Gastric cancer type } & \multicolumn{2}{|c|}{ MUC2 } & \multicolumn{2}{|c|}{$M U C 5 A C$} & \multicolumn{2}{|c|}{ MUC6 } & \multicolumn{2}{|c|}{$C D X 2$} \\
\hline & & & $\begin{array}{c}\text { Positive cases } \\
(\%)\end{array}$ & Score ${ }^{\mathrm{a}}$ & $\begin{array}{c}\text { Positive cases } \\
\text { (\%) }\end{array}$ & Score ${ }^{\mathrm{a}}$ & $\begin{array}{c}\text { Positive cases } \\
(\%)\end{array}$ & Score ${ }^{\mathrm{a}}$ & $\begin{array}{c}\text { Positive cases } \\
(\%)\end{array}$ & Score ${ }^{\mathrm{a}}$ \\
\hline \multirow[t]{4}{*}{ Intestinal type } & Tota & $\left.45^{\mathrm{b}}\right)$ & $23(51 \%)^{*}$ & $3.24 \pm 0.49^{*}$ & $23(51 \%)$ & $3.47 \pm 0.53$ & $18(40 \%)$ & $2.22 \pm 0.42$ & $30(67 \%)$ & $3.20 \pm 0.39$ \\
\hline & Differentiation & Well (14) & $10(71 \%)$ & $4.57 \pm 0.85$ & $7(50 \%)$ & $3.36 \pm 0.96$ & $5(36 \%)$ & $2.07 \pm 0.79$ & $11(79 \%)$ & $4.00 \pm 0.69$ \\
\hline & & Moderate (11) & $4(36 \%)$ & $2.09 \pm 0.88$ & $7(64 \%)$ & $4.27 \pm 1.05$ & $5(45 \%)$ & $2.36 \pm 0.82$ & $6(55 \%)$ & $2.27 \pm 0.73$ \\
\hline & & Poor (20) & $9(45 \%)$ & $2.95 \pm 0.77$ & $9(45 \%)$ & $3.10 \pm 0.81$ & $8(40 \%)$ & $2.25 \pm 0.64$ & $13(65 \%)$ & $3.15 \pm 0.61$ \\
\hline Diffuse type (19) & & & $11(58 \%)$ & $3.95 \pm 0.82$ & $14(74 \%)$ & $5.42 \pm 0.79$ & $8(42 \%)$ & $2.63 \pm 0.75$ & $8(42 \%)$ & $2.37 \pm 0.68$ \\
\hline Mucinous type (6) & & & $6(100 \%)^{*}$ & $7.17 \pm 0.54^{*}$ & $3(50 \%)$ & $3.83 \pm 1.72$ & $0(0 \%)$ & $0 \pm 0$ & $5(83 \%)$ & $4.33 \pm 0.99$ \\
\hline Total (70) & & & $40(57 \%)$ & $3.77 \pm 0.41$ & $40(57 \%)$ & $4.03 \pm 0.43$ & $26(37 \%)$ & $2.14 \pm 0.34$ & $43(61 \%)$ & $3.07 \pm 0.33$ \\
\hline
\end{tabular}

${ }^{\mathrm{a}}$ Scores were expressed as the mean \pm s.e.m.

${ }^{\mathrm{b}}$ The figures in parentheses in this column are the numbers of cases.

${ }^{*} P<0.05$ when compared with the other in the same column.
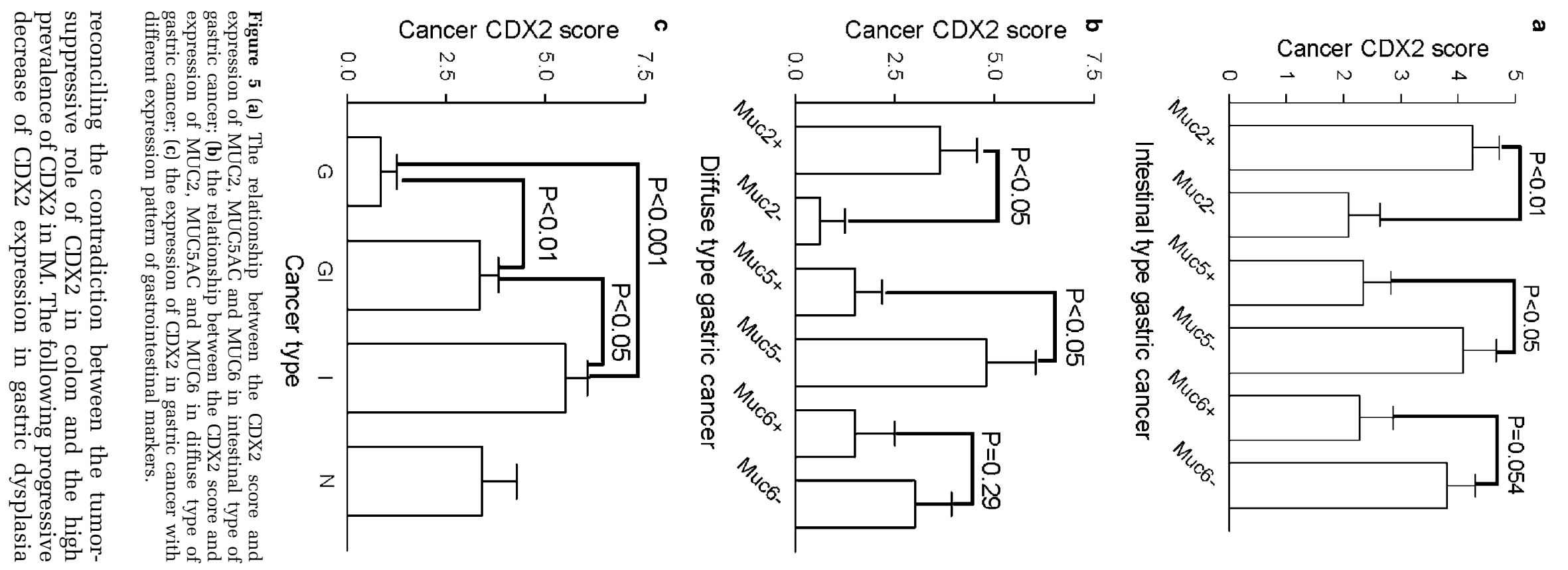

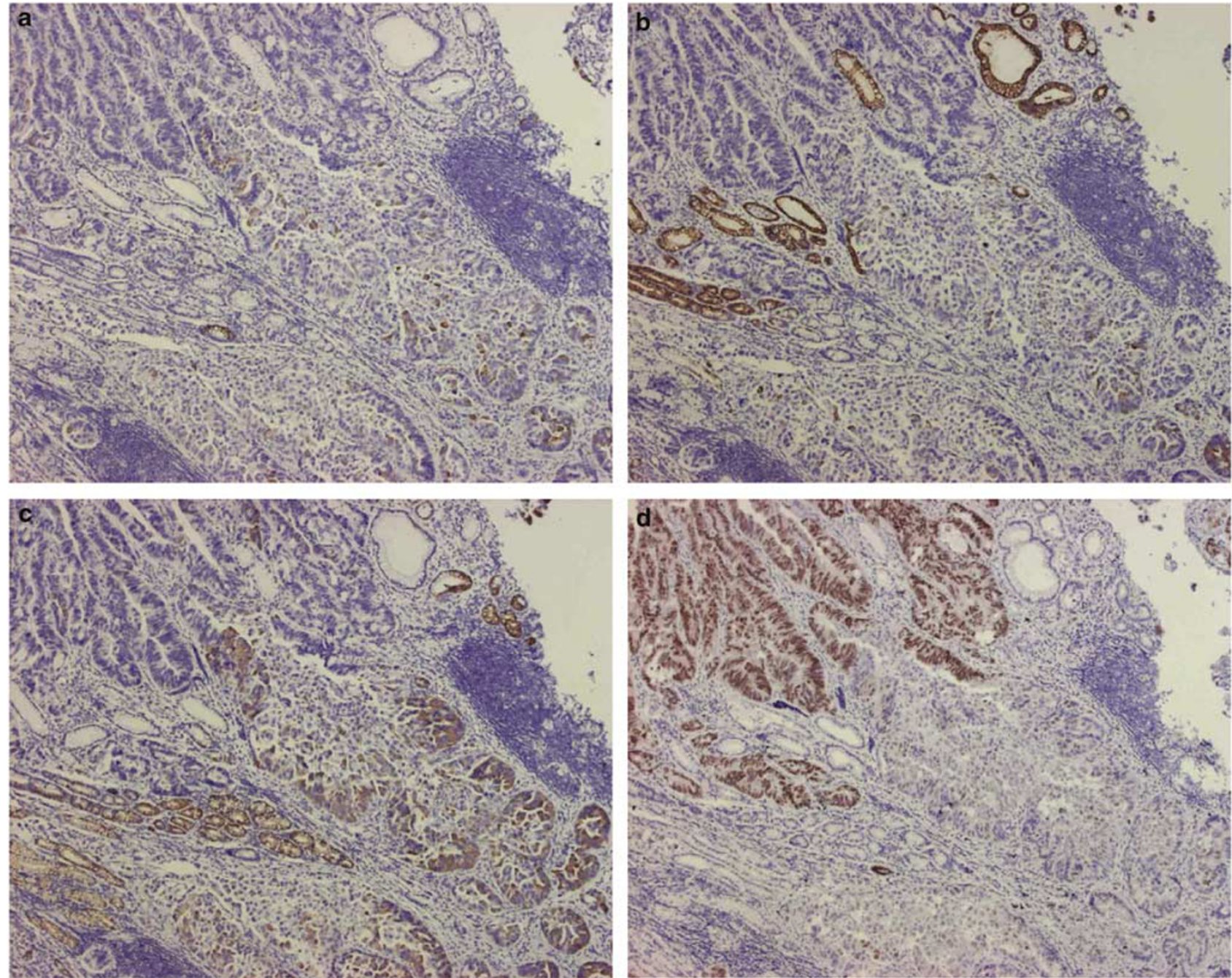

Figure 6 Immunohistochemistry staining of MUC2 (a) MUC5AC (b) MUC6 (c) CDX2 (d) in a case of gastric cancer. The left upper part of gastric cancer cells with strong CDX2 expression expressed less gastric mucins (MUC5AC and MUC6) than the right lower part of cancer cells with weak CDX2 expression.

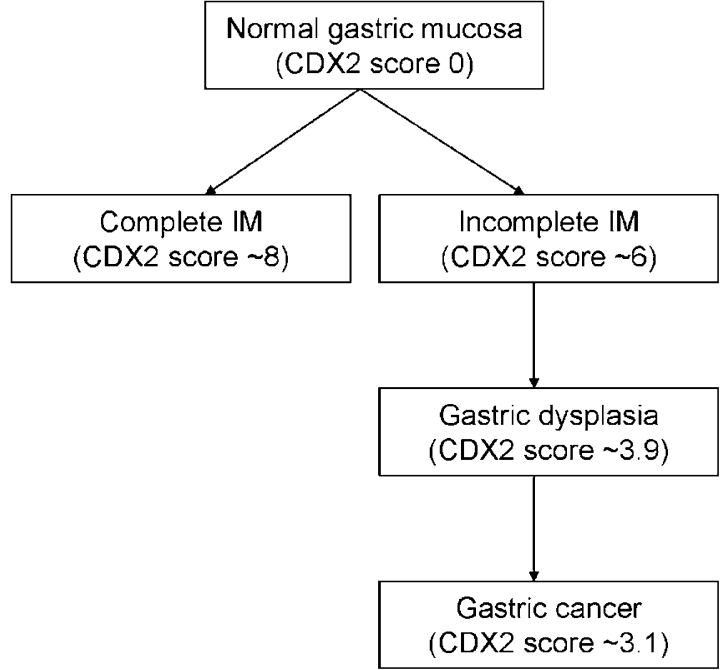

Figure 7 The expression of CDX2 is initially increased in IM and then progressively decreased along Correa's cascade of gastric carcinogenesis. and gastric cancer suggests that CDX2 plays a similar anticarcinogenetic role in gastric and colorectal carcinogenesis. IM or dysplasia with low expression of CDX2 may be useful markers for higher risk of gastric cancer.

\section{Acknowledgement}

Agency for Science, Technology and Research, Singapore, and Human Frontier Science Program RGP0375/ 2001-M202. Q Liu and KG Yeoh received funding support from SCS Grants GN-15 \& MN-05 awarded by the Singapore Cancer Syndicate, Agency for Science, Technology and Research, Singapore.

\section{References}

1 Correa P. Human gastric carcinogenesis: a multistep and multifactorial process-First American Cancer 
Society Award Lecture on Cancer Epidemiology and Prevention. Cancer Res 1992;52:6735-6740.

2 Meining A, Morgner A, Miehlke S, et al. Atrophymetaplasia-dysplasia-carcinoma sequence in the stomach: a reality or merely a hypothesis? Best Pract Res Clin Gastroenterol 2001;15:983-998.

3 Hattori T. Development of adenocarcinomas in the stomach. Cancer 1986;57:1528-1534.

4 Yuasa Y. Control of gut differentiation and intestinaltype gastric carcinogenesis. Nat Rev Cancer 2003;3: 592-600.

5 Filipe MI, Potet F, Bogomoletz WV, et al. Incomplete sulphomucin-secreting intestinal metaplasia for gastric cancer. Preliminary data from a prospective study from three centres. Gut 1985;26:1319-1326.

6 Zerbib F, Lenk C, Sawan B, et al. Long-term effects of Helicobacter pylori eradication on gastric antral mucosa in duodenal ulcer patients. Eur J Gastroenterol Hepatol 2000;12:719-725.

7 Matsukura N, Suzuki K, Kawachi T, et al. Distribution of marker enzymes and mucin in intestinal metaplasia in human stomach and relation to complete and incomplete types of intestinal metaplasia to minute gastric carcinomas. J Natl Cancer Inst 1980;65:231-240.

8 Jass JR, Filipe MI. Sulphomucins and precancerous lesions of the human stomach. Histopathology 1980;4: 271-279.

9 Filipe MI, Munoz N, Matko I, et al. Intestinal metaplasia types and the risk of gastric cancer: a cohort study in Slovenia. Int J Cancer 1994;57:324-329.

10 Rokkas T, Filipe MI, Sladen GE. Detection of an increased incidence of early gastric cancer in patients with intestinal metaplasia type III who are closely followed up. Gut 1991;32:1110-1113.

$11 \mathrm{Wu}$ MS, Shun CT, Lee WC, et al. Gastric cancer risk in relation to Helicobacter pylori infection and subtypes of intestinal metaplasia. Br J Cancer 1998;78: 125-128.

12 Ramesar KC, Sanders DS, Hopwood D. Limited value of type III intestinal metaplasia in predicting risk of gastric carcinoma. J Clin Pathol 1987;40:1287-1290.

13 El-Zimaity HM, Ramchatesingh J, Saeed MA, et al. Gastric intestinal metaplasia: subtypes and natural history. J Clin Pathol 2001;54:679-683.

14 Smith JL, Dixon MF. Is subtyping of intestinal metaplasia in the upper gastrointestinal tract a worthwhile exercise? An evaluation of current mucin histochemical stains. Br J Biomed Sci 2003;60: 180-186.

15 Witek ME, Nielsen K, Walters R, et al. The putative tumor suppressor Cdx2 is overexpressed by human colorectal adenocarcinomas. Clin Cancer Res 2005;11: 8549-8556.

16 Ee HC, Erler T, Bhathal PS, et al. Cdx-2 homeodomain protein expression in human and rat colorectal adenoma and carcinoma. Am J Pathol 1995;147: 586-592.

17 Mallo GV, Rechreche H, Frigerio JM, et al. Molecular cloning, sequencing and expression of the mRNA encoding human Cdx1 and Cdx2 homeobox. Downregulation of $\mathrm{Cdx} 1$ and $\mathrm{Cdx} 2$ mRNA expression during colorectal carcinogenesis. Int J Cancer 1997;74: 35-44.

18 Choi BJ, Kim CJ, Cho YG, et al. Altered expression of CDX2 in colorectal cancers. APMIS 2006;114:50-54.

19 Mallo GV, Soubeyran P, Lissitzky JC, et al. Expression of the Cdx1 and Cdx2 homeotic genes leads to reduced malignancy in colon cancer-derived cells. J Biol Chem 1998;273:14030-14036.

20 Gendron FP, Mongrain S, Laprise P, et al. The CDX2 transcription factor regulates furin expression during intestinal epithelial cell differentiation. Am J Physiol Gastrointest Liver Physiol 2006;290:G310-G318.

21 Aoki K, Tamai Y, Horiike S, et al. Colonic polyposis caused by mTOR-mediated chromosomal instability in Apc+/Delta716 Cdx2+/- compound mutant mice. Nat Genet 2003;35:323-330.

22 Bonhomme C, Duluc I, Martin E, et al. The Cdx2 homeobox gene has a tumour suppressor function in the distal colon in addition to a homeotic role during gut development. Gut 2003;52:1465-1471.

23 Silberg DG, Sullivan J, Kang E, et al. Cdx2 ectopic expression induces gastric intestinal metaplasia in transgenic mice. Gastroenterology 2002;122:689-696.

24 Mutoh H, Hakamata Y, Sato K, et al. Conversion of gastric mucosa to intestinal metaplasia in Cdx2expressing transgenic mice. Biochem Biophys Res Commun 2002;294:470-479.

25 Mutoh H, Sakurai S, Satoh K, et al. Development of gastric carcinoma from intestinal metaplasia in Cdx2transgenic mice. Cancer Res 2004;64:7740-7747.

26 Almeida R, Silva E, Santos-Silva F, et al. Expression of intestine-specific transcription factors, CDX1 and CDX2, in intestinal metaplasia and gastric carcinomas. J Pathol 2003;199:36-40.

27 Bai YQ, Yamamoto H, Akiyama Y, et al. Ectopic expression of homeodomain protein CDX2 in intestinal metaplasia and carcinomas of the stomach. Cancer Lett 2002;176:47-55.

28 Fan Z, Li J, Dong B, et al. Expression of Cdx2 and hepatocyte antigen in gastric carcinoma: correlation with histologic type and implications for prognosis. Clin Cancer Res 2005;11:6162-6170.

29 Roessler K, Monig SP, Schneider PM, et al. Coexpression of CDX2 and MUC2 in gastric carcinomas: correlations with clinico-pathological parameters and prognosis. World J Gastroenterol 2005;11:3182-3188.

30 Seno H, Oshima M, Taniguchi MA, et al. CDX2 expression in the stomach with intestinal metaplasia and intestinal-type cancer: Prognostic implications. Int J Oncol 2002;21:769-774.

31 Dang LH, Chen F, Knock SA, et al. CDX2 does not suppress tumorigenicity in the human gastric cancer cell line MKN45. Oncogene 2005;25:2048-2059.

32 Mizoshita T, Tsukamoto T, Nakanishi H, et al. Expression of Cdx2 and the phenotype of advanced gastric cancers: relationship with prognosis. J Cancer Res Clin Oncol 2003;129:727-734.

33 Reis CA, David L, Correa P, et al. Intestinal metaplasia of human stomach displays distinct patterns of mucin (MUC1, MUC2, MUC5AC, and MUC6) expression. Cancer Res 1999;59:1003-1007.

34 Rocha AS, Soares P, Machado JC, et al. Mucoepidermoid carcinoma of the thyroid: a tumour histotype characterised by P-cadherin neoexpression and marked abnormalities of E-cadherin/catenins complex. Virchows Arch 2002;440:498-504.

35 Tatematsu M, Tsukamoto T, Inada K. Stem cells and gastric cancer: role of gastric and intestinal mixed intestinal metaplasia. Cancer Sci 2003;94:135-141.

36 Mizoshita T, Tsukamoto T, Tanaka H, et al. Colonic and small-intestinal phenotypes in gastric cancers: relationships with clinicopathological findings. Pathol Int 2005;55:611-618. 
37 Leung WK, Sung JJ. Review article: intestinal metaplasia and gastric carcinogenesis. Aliment Pharmacol Ther 2002;16:1209-1216.

38 Debruyne PR, Witek M, Gong L, et al. Bile acids induce ectopic expression of intestinal guanylyl cyclase $\mathrm{C}$ Through nuclear factor-kappaB and Cdx2 in human esophageal cells. Gastroenterology 2006;130:1191-1206.

39 Bai Y, Akiyama Y, Nagasaki H, et al. Distinct expression of CDX2 and GATA4/5, development-related genes, in human gastric cancer cell lines. Mol Carcinog 2000;28:184-188.

40 Yagi OK, Akiyama Y, Yuasa Y. Genomic structure and alterations of homeobox gene CDX2 in colorectal carcinomas. Br J Cancer 1999;79:440-444.
41 Lorentz O, Cadoret A, Duluc I, et al. Downregulation of the colon tumour-suppressor homeobox gene Cdx-2 by oncogenic ras. Oncogene 1999;18:87-92.

42 Yuasa Y, Nagasaki H, Akiyama Y, et al. Relationship between CDX2 gene methylation and dietary factors in gastric cancer patients. Carcinogenesis 2005;26: 193-200.

43 Kawai H, Tomii K, Toyooka S, et al. Promoter methylation downregulates CDX2 expression in colorectal carcinomas. Oncol Rep 2005;13:547-551.

44 Carneiro F, Moutinho-Ribeiro M, David L, et al. Signet ring cell carcinoma of the stomach: a morphometric, ultrastructural, and DNA cytometric study. Ultrastruct Pathol 1992;16:603-614. 